\title{
Cosmic Ray Energetics And Mass for the International Space Station (ISS-CREAM)
}

E. S. Seo ${ }^{1,2, *}$, Y. Amare ${ }^{1}$, D. Angelaszek ${ }^{1,2}$, N. Anthony ${ }^{1}$, G. H. Choi ${ }^{3}$, M. Chung ${ }^{1}$, M. Copley $^{1}$, L. Derome ${ }^{4}$, L. Eraud ${ }^{4}$, C. Falana1, A. Gerrety ${ }^{1}$, L. Hagenau ${ }^{1}$, J. H. Han ${ }^{1}$, H. G. Huh", Y. S. Hwang ${ }^{5}$, H. J. Hyun ${ }^{5}$, H.B. Jeon ${ }^{5}$, J. A. Jeon ${ }^{3}$, S. Jeong ${ }^{3}$, S. C. Kang, ${ }^{5}$

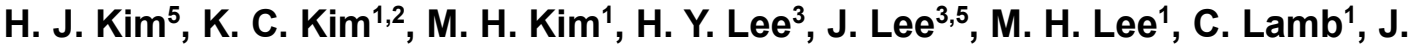
Liang$^{1}$, L. Lu1 ${ }^{1}$, J. P. Lundquist ${ }^{1,3}$, L. Lutz ${ }^{1}$, B. Mark ${ }^{1}$, A. Menchaca-Rocha ${ }^{6}$, T. Mernik', M. Nester ${ }^{1}$, O. Ofoha ${ }^{1}$, H. Park ${ }^{5}$, I. H. Park ${ }^{3}$, J. M. Park ${ }^{5}$, N. PicotClemente $^{1}$, S. Rostsky ${ }^{1}$, J. R. Smith ${ }^{1}$, R. Takeishi ${ }^{3}$, T. Tatoli ${ }^{1}$, P. Walpole ${ }^{1}$, R. P. Weinmann ${ }^{1}$, J. $\mathbf{W u}^{1}$, Z. Yin ${ }^{1,2}$, Y. S. Yoon ${ }^{1,2}$ and H. G. Zhang ${ }^{1}$

${ }^{I}$ Inst. for Phys. Sci. and Tech., University of Maryland, College Park, MD, USA

${ }^{2}$ Dept. of Physics, University of Maryland, College Park, MD, USA

${ }^{3}$ Dept. of Physics, Sungkyunkwan University, Republic of Korea

${ }^{4}$ Laboratoire de Physique Subatomique et de Cosmologie, Grenoble, France

${ }^{5}$ Dept. of Physics, Kyungpook National University, Republic of Korea

${ }^{6}$ Instituto de Fisica, Universidad Nacional Autonoma de Mexico, Mexico

The ISS-CREAM payload was launched on the SpaceX-12 Commercial Resupply Service mission to the International Space Station (ISS) from NASA's Kennedy Space Center on August 14, 2017. It was successfully installed and activated on the ISS Japanese Experiment Module Exposed Facility as an attached payload on August 22, 2017. The ISS-CREAM instrument is configured with complementary particle detectors capable of measuring elemental spectra for $\mathrm{Z}$ $=1-26$ nuclei in the energy range $\sim 10^{12}-10^{15} \mathrm{eV}$; as well as electrons at multi-TeV energies. The four layers of its finely segmented Silicon Charge Detectors provide precise charge measurements, and its ionization Calorimeter provides energy measurements. In addition, scintillator-based Top and Bottom Counting Detectors and a Boronated Scintillator Detector distinguish electrons from nuclei. The goal is to understand cosmic ray origin, acceleration and propagation by extending direct measurements of cosmic rays to the highest practical energy. Onorbit performance of the instrument and preliminary results from the ongoing analysis are presented.

36th International Cosmic Ray Conference -ICRC2019.

July 24th - August 1st, 2019

Madison, WI, U.S.A.

${ }^{1}$ Speaker for the ISS-CREAM Collaboration ${ }^{\#}$

*E-mail: seo@umd.edu

\# For the collaboration list see PoS(ICRC2019)1177 


\section{Introduction}

The Cosmic Ray Energetics And Mass (CREAM) experiment was initially developed to measure cosmic ray elemental spectra using a series of balloon flights [1]. The balloon-borne CREAM was flown seven times (12/15/04 - 12/28/16) over Antarctica accumulating 191 days of flight time, the longest exposure for a single balloon project. The instrument integrated for CREAM-VII was renamed Boron And Carbon Cosmic rays in the Upper Stratosphere (BACCUS) to distinguish it from the International Space Station (ISS) mission.

Building on the success of the balloon flights, the payload was transformed for accommodation on the ISS, specifically, NASA's share of the Japanese Experiment Module Exposed Facility (JEM-EF). This version of CREAM, aka ISS-CREAM, was launched aboard a SpaceX Falcon 9 rocket as part of the 12th Commercial Resupply Service (CRS-12) mission to the ISS on August 14, 2017 after 2 years of storage at NASA Kennedy Space Center in Cape Canaveral, Florida. The CRS-12 mission, aka SpX-12, carried $\sim 6,000$ pounds of scientific research, crew supplies and hardware to the ISS in support of the Expedition 52 and 53 crew members including the 1.4 ton $\left(1.85 \times 0.95 \times 1 \mathrm{~m}^{3}\right)$ ISS-CREAM payload [2]. Two days after launch, the Dragon spacecraft with ISS-CREAM in the unpressurized trunk arrived at the ISS. The ISS-CREAM payload was extracted from the trunk by the Space Station Remote Manipulator System (SSRMS) on August 21 and transferred to the Japanese Experiment Module Remote Manipulator System (JEMRMS). Following the installation of ISS-CREAM on the ISS JEMEFU\#2 (see Fig. 1), ISS-CREAM was activated successfully on August 22, 2017. The launch, termination dates, and the duration for each flight are summarized in Table 1.

The goal of this experiment is to understand the origin, acceleration, and galactic propagation of the bulk of cosmic rays by extending direct measurements of cosmic-ray composition to energies capable of generating gigantic air showers that have mainly been observed from the ground. Precise measurements of the energy dependence of elemental spectra at these high energies, where the rigidity-dependent supernova acceleration limit could be reflected in a composition change, provide a key to understanding cosmic ray acceleration and propagation. Specifically, it would (1) determine how the observed spectral differences of protons

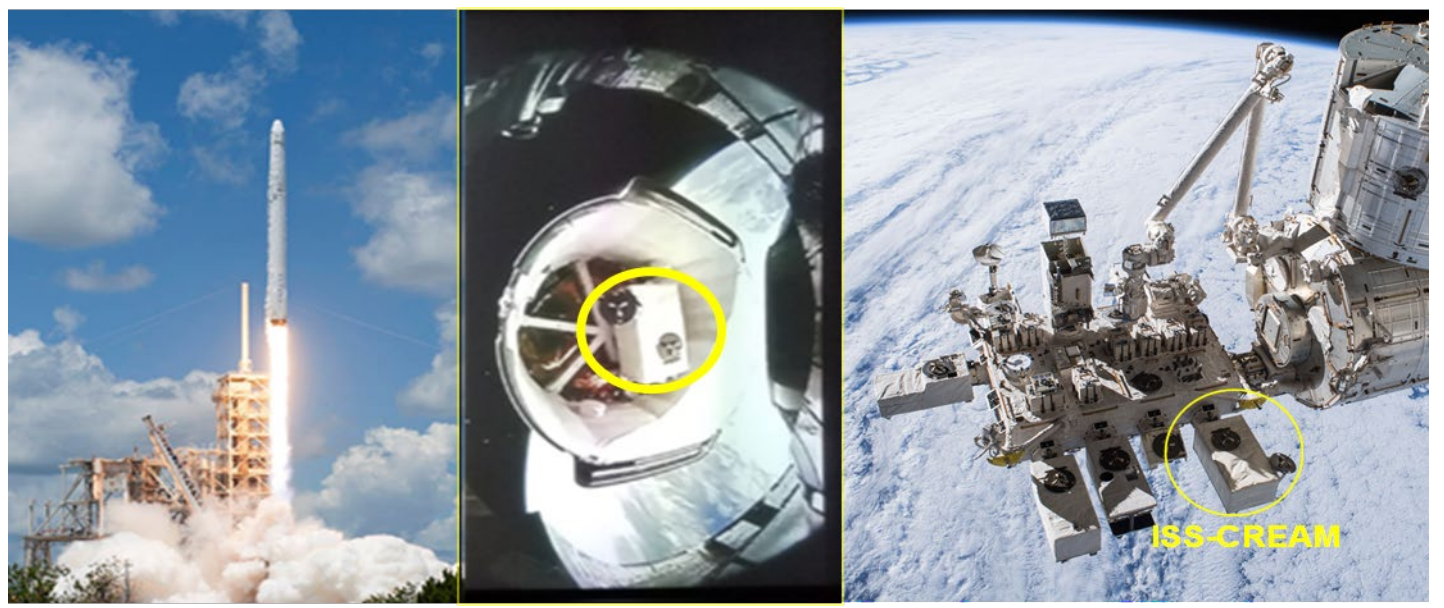

Fig. 1. Left, the SpaceX-12 launch; middle, the ISS-CREAM payload in the Dragon spacecraft trunk when the spacecraft separated from the upper stage of the Falcon rocket; right, the ISS-CREAM payload on the ISS JEM-EF\#2. The ISS-CREAM payload is marked with a yellow circle. 
and heavier nuclei evolve at higher energies approaching the "knee"; (2) be capable of measuring potential changes in the spectra of secondary nuclei resulting from the interactions of primary cosmic rays with the interstellar medium; (3) conduct a sensitive search for spectral features, such as a bend in the proton spectrum; and (4) measure electrons with sufficient accuracy to determine whether or not a nearby cosmic source exists.

Table 1. Summary of CREAM flights.

\begin{tabular}{|l|lll|}
\hline & Launch & Termination & Duration \\
\hline CREAM-I & 2004.12 .16 & 2005.1 .27 & 41 days $22 \mathrm{hrs}$ \\
\hline CREAM-II & 2005.12 .15 & 2006.1 .13 & 28 days $10 \mathrm{hrs}$ \\
\hline CREAM-III & 2007.12 .19 & 2008.1 .17 & 28 days $22 \mathrm{hrs}$ \\
\hline CREAM-IV & 2008.12 .18 & 2009.1 .7 & 19 days $13 \mathrm{hrs}$ \\
\hline CREAM-V & 2009.12 .1 & 2010.1 .8 & 37 days $10 \mathrm{hrs}$ \\
CREAM-VI & 2010.12 .21 & 2010.12 .26 & 5 days $16 \mathrm{hrs}$ \\
\hline BACCUS & 2016.11 .28 & 2016.12 .28 & 30 days $2 \mathrm{hrs}$ \\
\hline ISS-CREAM & 2017.8 .14 & 2019.2 .12 & 546 days \\
\hline
\end{tabular}

\section{Instrument}

The ISS-CREAM instrument is configured with complementary particle detectors to determine the charge and energy of high-energy cosmic rays from protons to iron with excellent resolution. It is designed to meet the challenging requirement to have a large enough geometry factor to collect adequate statistics for the low flux of high-energy particles, and yet stay within the JEM-EF weight and volume limit. The primary instrument flown on the balloon-borne CREAM [3] was repackaged into the smaller volume available on the JEM-EF [4,5].

As shown in Fig. 2 left, the ISS-CREAM instrument consists of the tungsten/scintillator sampling calorimeter (CAL) including carbon targets previously flown on balloons for energy measurements, and four layers of a finely segmented Silicon Charge Detector (SCD) for charge measurements. They have already demonstrated their capabilities of determining the charge and energy of high-energy cosmic rays for the proton to iron elemental range with excellent resolution [6]. In addition, the Top and Bottom Counting Detectors (TCD/BCD) located above and below the CAL, respectively, help electron measurements by using different shower profiles to distinguish electrons from protons $[7,8]$. They also provide a low energy trigger while the CAL provides a high energy trigger. The Boronated Scintillator Detector (BSD) located at the bottom of the instrument provides additional e/p separation by measuring late thermal neutron shower activity [9].

The calorimeter (CAL) combines $0.5 \lambda_{\text {int }}$ thick graphite targets and a stack of 20 tungsten plates, each $50 \mathrm{~cm} \times 50 \mathrm{~cm} \times 3.5 \mathrm{~mm}\left(1 \mathrm{X}_{0}\right)$ thick, followed by a layer of $0.5 \mathrm{~mm}$ diameter scintillating fibers grouped into fifty $1 \mathrm{~cm}$-wide ribbons [10]. The carbon target induces hadronic interactions so showers develop in the CAL. To cover a wide dynamic range, three (low, mid, and high) readout ranges are used via the optical division described in [3]. The CAL module has been calibrated in a series of beam tests at the CERN Super Proton Synchotron (SPS), which provides the highest-energy test-beam particles available [11-13]. Energy deposition in the CAL determines the particle energy and provides tracking information to determine which segment(s) 
of the charge detectors to use for the charge measurement. Tracking for showers is accomplished by extrapolating each shower axis back to the charge detectors $[14,15]$.

It is important for the charge detectors to be segmented as small as possible to minimize hits of accompanying backscattered particles in the same segment as the incident particle to avoid its charge misidentificaiton [16, 17]. The ISS-CREAM SCD is comprised of an array of DC-type silicon PIN diodes. Each of the four SCD layers covers an $78 \times 74 \mathrm{~cm}^{2}$ area. A cosmic ray passing through the sensor produces ionization in the depleted region that is proportional to the square of the particle charge. The $1.55 \times 1.38 \mathrm{~cm}^{2}$ active area of each pixel is optimized to reduce the effect of backscatter from showers in the calorimeter, while keeping the channel count and power at manageable levels. The readout electronics are designed around a 16-channel CR-1.4A ASIC for each sensor followed by 16-bit ADCs. This allows fine charge resolution over a wide dynamic range covering up to $Z=26$ signals [18].

The TCD and BCD comprise $50 \times 50 \times 0.5 \mathrm{~cm}^{3}$ and $60 \times 60 \times 1 \mathrm{~cm}^{3}$ plastic scintillators, respectively. The light produced in each detector is measured by a $20 \times 20$ photodiode array. Details of the detector design and performance are summarized in [19]. At least one hit in each of TCD and BCD provides a coincidence trigger.

Two classes of events are of interest for ISS-CREAM: high-energy shower events, regardless of the incident particle charge, and events with high-charge incident nuclei, whether or not they interacted and generated a shower in the calorimeter. The trigger system designed to initiate data collection for both is made of a Master Trigger, with inputs from the two detector trigger systems, the calorimeter trigger and TCD/BCD trigger. The calorimeter trigger is designed for high energy shower events by requiring significant signals in a number of consecutive layers (changeable by commands but typically six was used in flight), while the TCD/BCD trigger is designed for events in which a high-charge nucleus traverses the instrument with or without interactions.

\section{Data Analysis and On-orbit Performance}

As part of the extensive development program, the CREAM sub-detectors were tested and calibrated at CERN's SPS accelerator facility, proving that the detectors work as designed, and showing that the simulation correctly describes them, allowing extrapolation to the energy range of interest [11-13, 20-22]. The in-flight calibration included periodic charge injection events, LED flashes, and pedestal runs to provide the information needed to correct for electronic pedestals, as well as gain changes as a function of time, e.g., due to changes in temperature, etc.

For events selected with a science trigger, the trajectory of each event was reconstructed from a linear fit to the core of the shower axis through the multiple layers of scintillating fiber strips in CAL. This reconstructed trajectory is required to traverse the SCD active area. Figure 2 (right) shows a cosmic ray event from the flight data illustrating the reconstructed trajectory that is consistent with the hit positions in the SCD, while Fig. 2 (middle) illustrates a shower development in the instrument using Monte Carlo simulations [23]. The particle energy is determined from ionization energy deposits of cascades developed in the calorimeter. Details of the CAL on-orbit performance is reported in [24].

Figure 3 left shows the measured calorimeter energy deposit for various trigger conditions. The red line represents the high energy trigger from the CAL. The pink and green lines represent triggers from the $\mathrm{TCD} / \mathrm{BCD}$, where the pink line corresponds to the low energy trigger. As illustrated with 


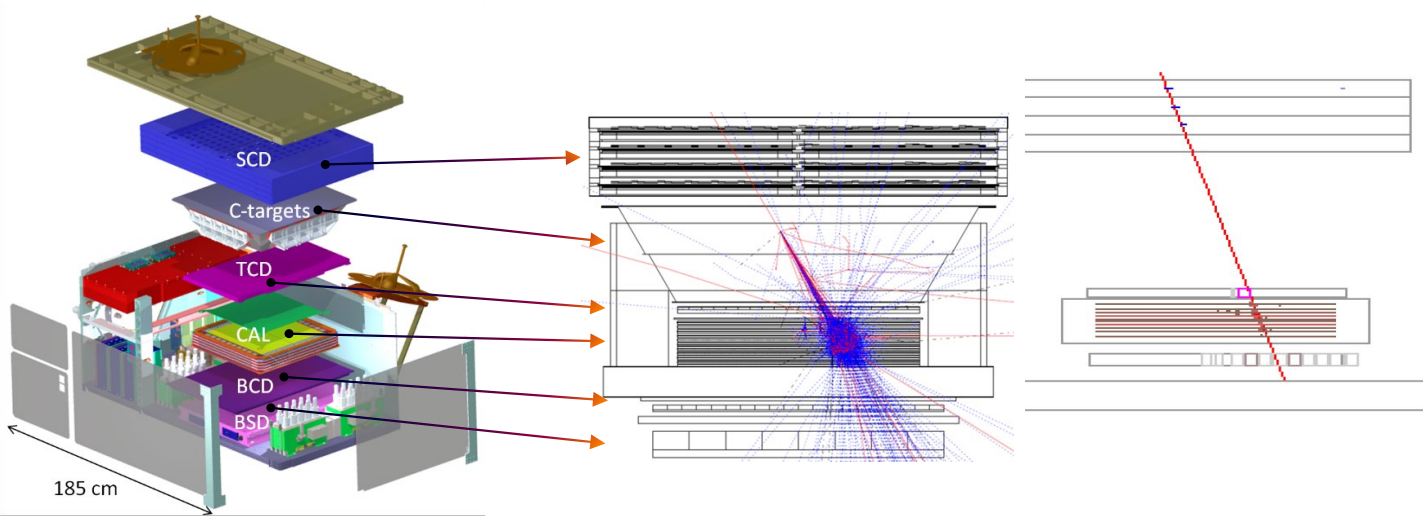

Figure 2. An exploded view of the ISS-CREAM instrument (left), an example of cosmic ray event illustrating a shower development in the instrument using Monte Carlo simulations (middle), an actual cosmic ray event from the flight data (right).

the thick black line, the measured calorimeter energy deposit distribution shows a reasonable power law. With a very preliminary calibration based only on the low range readout, all-particle counts are shown as a function of incident energy in Fig. 3 right. The ISS-CREAM data (red symbols) are compared with CREAM-III balloon flight data (blue symbols), with a normalization at $10 \mathrm{TeV}$. This plot is not intended for spectral index or absolute flux measurements, but rather for a quick consistency check.

Corrections are still to be made for channel to channel variation of electronics, energy dependent shower leakage for the energy scale, and charge dependent efficiencies. Flight data can be used offline to further calibrate the detectors. Shower event data will allow inter-range calibration between the low-, mid-, and high-energy ranges in the calorimeter. Since the measured spectra are convolutions of the input spectra and the inherent resolution, the true input spectra could be obtaind by deconvolution to correct for the spillover among the energy bins due to the finite energy
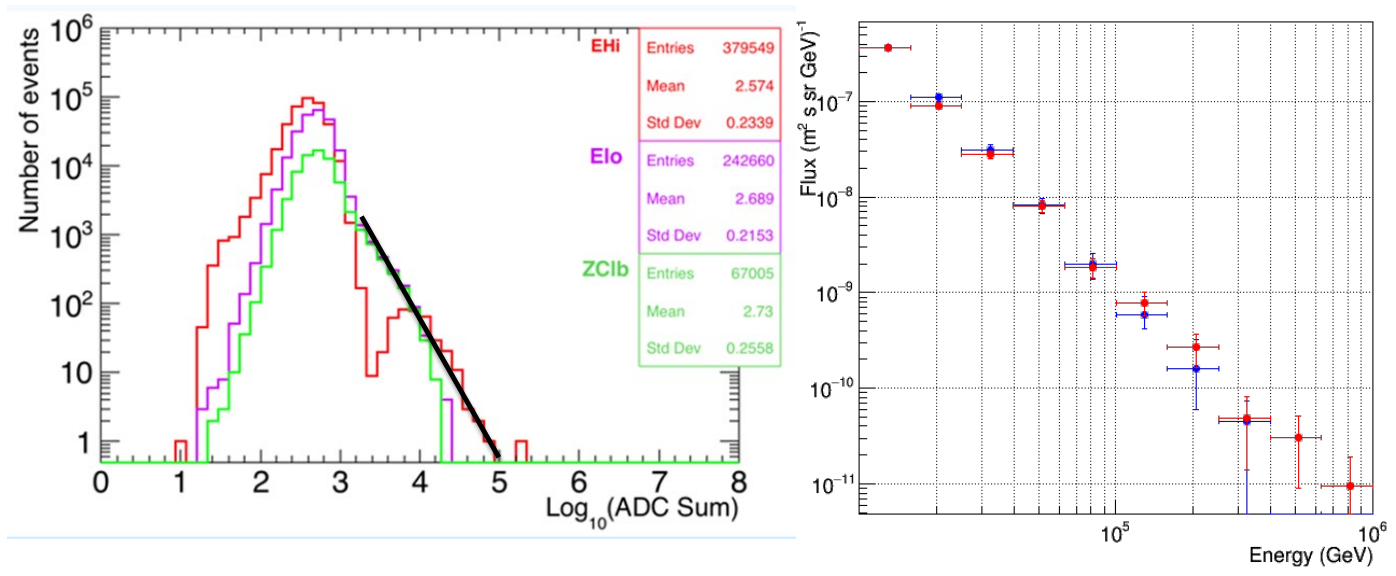

Figure 3. The CAL energy deposit distribution for various trigger conditions shows a clear power law behavior of cosmic ray data (left) even before all the corrections are applied. Preliminary all-particle counts as a function of incident energy (red symbols) are compared with CREAM-III balloon flight data (blue symbols), with a normalization at $10 \mathrm{TeV}$. Note corrections considering electronic channel to channel variations are still to be made. 
resolution. The measured spectra will have to be corrected for the geometry factor, live time and efficiencies to determine absolute fluxes. More details of the on-going analysis can be found in [25].

The distribution of cosmic-ray elements measured with the SCD is shown in Fig. 4. The charge peaks for major elements from $\mathrm{Z}=1$ to 26 are clearly identified with its $\sigma \sim 0.2 \mathrm{e}$ charge resolution and good linearity. The relative abundance in this plot has no physical significance, because needed corrections for interactions and propagations have not been applied to these data. The charge resolution will improve as the tracking algorithm improves by including the TCD/BCD in the current CAL track reconstruction, as well as including various corrections such as channel to channel variations [26].

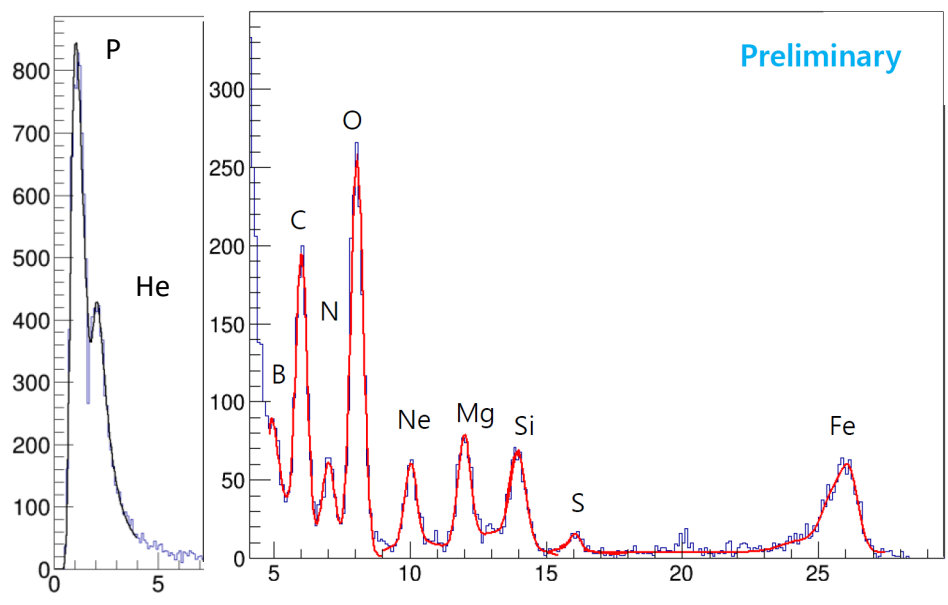

Figure 4: Preliminary flight data: Distribution of cosmic-ray charge measured with the SCD. The charge reconstructed for a fraction of the flight data is shown in units of the elementary charge $e$. The individual elements are clearly identified. The relative abundance in this plot has no physical significance, because needed corrections for interactions and propagations have not been applied to these data.

\section{Conclusion}

Since its installation on the ISS on August 22, 2017, the ISS-CREAM experiment collected data until it was shut down on February 12, 2019. The Science Operations Center (SOC) at the University of Maryland successfully sent commands to and received data from the Science Flight Computer (SFC) on board ISS-CREAM. The SOC's operation was coordinated with the Huntsville Operations Support Center (HOSC)/Payload Operations Integration Center (POIC) at NASA's Marshall Space Flight Center for this operation. The Telescience Resources Kit was used for data/command path between HOSC and SOC. The Software Toolkit for Ethernet Lab-Like Architecture was used for the communications between the SOC and the ISS-CREAM payload interfacing with the CREAM Data Acquisition system (CDAQ) [27].

The instrument-interface unit on board the ISS-CREAM instrument successfully monitored the SFC's functional status using two watchdog timers, and took actions autonomously when it was needed, e.g., rebooting, selecting boot-source, and power switching between two SFC's. It was also possible to send CDAQ commands to ISS-CREAM through a timed commandable sequence called a time-liner, which made the SOC operation more autonomous [28]. 
The SOC processed the flight data and distributed the Level 0 output, written in ROOT format, to collaborators for further processing. The data processing included checking the dataset for structure and data-block defects, removal of flight-software "debug" records, time ordering of all records, as well as identifying and fixing any flight specific problems with the dataset. Engineering data were converted to appropriate units such as temperatures, voltages, currents, etc. The Level 1 and Level 2 data processing involve those who developed each detector. For processing the $\mathrm{L} 0$ data to $\mathrm{L} 1$, detailed calibration for each detector component was performed to convert the data into "physics units," i.e. energy deposits in each detector component. The higher level processing to L2, assigns the "best" values for incident charge, energy and trajectory. SOC facilitated the code integration with a Git server, a visualizing version control system. Data analysis is still in the early stage, but the preliminary data look promising. Refinement of the initial detector calibrations is in progress by applying gain corrections for mid and high ranges, channel by channel. Further corrections will be made to reflect the actual flight experience, which involve temperature or other time-dependent effects.

\section{Acknowledgements}

This work was supported in the U.S. by NASA grant NNX17AB41G, in Korea by National Research Foundation grants 2018R1A2A1A05022685 and 2018R1A6A1A06024970, and their predecessor grants. It was also supported in France by IN2P3/CNRS and CNES and in Mexico by DGAPA-UNAM project IN109617. The authors thank NASA GSFC WFF and its contractors for engineering support and project management, JSC ISS Program Office for the launch support and ISS accommodation, MSFC for the operational support, and KSC and SpaceX for the launch support.

\section{References}

[1] E. S. Seo et al., Cosmic-ray energetics and mass (CREAM) balloon project, Advances in Space Research, Volume 33, Issue 10, 1777-1785, 2004.

[2] E. S. Seo et al, Cosmic Ray Energetics And Mass for the International Space Station (ISS-CREAM), Advances in Space Research, 53/10 (2014) 1451.

[3] H. S. Ahn et al. The Cosmic Ray Energetics And Mass (CREAM) instrument, Nucl. Inst. Meth. A, 579 (2007) 1034 [http://dx.doi.org/10.1016/j.nima.2007.05.203].

[4] E. S. Seo et al., Cosmic Ray Energetics And Mass: from balloons to the ISS, PoS(ICRC2015)574, 2015.

[5] J.R. Smith et al., The Cosmic Ray Energetics And Mass for the International Space Station (ISSCREAM) Instrument, PoS(ICRC2017)199, 2017.

[6] H. S. Ahn et al., Discrepant Hardening Observed in Cosmic-ray Elemental Spectra, Astrophys. J. Lett. 714 (2010) L89 [doi:10.1088/2041-8205/714/1/L89].

[7] S. Kang et al. e/p separation study using the ISS-CREAM Top and Bottom Counting Detectors, submitted to PoS(ICRC2019).

[8] J. M. Park et al. A simulation study of Top and Bottom Counting Detectors in ISS-CREAM experiment for cosmic ray electron physics, Advances in Space Research, 62/10, 2939-2944, 2018. 
[9] J. T. Link et al. Design and Performance of the ISS-CREAM Boronated Scintillator Detector, PoS(ICRC2015)611, 2015.

[10] M. H. Lee et al., Performance of the CREAM-III calorimeter, IEEE Transactions on Nuclear Science, 56, 1396-1399, 2009.

[11] N. Picot-Clemente et al., Performance of the ISS-CREAM Calorimeter, PoS(ICRC2017)247, 2017.

[12] J.H. Han et al., Calibration of the CREAM calorimeter with beam test data, Proc. 32nd Int. Cosmic Ray Conf., Beijing, 6, 392, 2011.

[13] H. S. Ahn et al., Performance of CREAM Calorimeter: Results of Beam Tests, Nuclear Physics B(Poc. Suppl.), 150, 272-275, 2006.

[14] Y. S. Yoon et al., Proton and Helium Spectra from the CREAM-III Fight, Astrophys. J., 839:5, 2017.

[15] J. P. Lundquist, Track Reconstruction for ISS-CREAM Resulting in Improved Energy and Charge Resolutions, submitted to PoS(ICRC2019).

[16] O. Ganel and E.S. Seo, Improving cosmic-ray composition determination through better tracking, Advances in Space Research 26/11, 1835, 2000.

[17] O. Ganel, E.S. Seo, J.Z. Wang and J. Wu, A guide for ACCESS design considerations, Proceedings of STAIF - 1999, Ed. M.S. El-Genk, AIP, 458, 272-277, 1999.

[18] J. Lee et al. The ISS-CREAM Silicon Charge Detector for identification of the charge of cosmic rays up to $Z=26$ : design, fabrication and ground-test performance, Astropart. Phys., 112, 8-15, 2019.

[19] H.J. Hyun et al. Performances of photodiode detectors for top and bottom counting detectors of ISSCREAM experiment, NIM A 787, 134-139, 2015.

[20] N. H. Park et al., Beam test of a dual layer silicon charge detector (SCD) for the CREAM experiment, Nucl. Instrum. Methods A, 581, 133-135, 2007

[21] I.H. Park et al., Heavy ion beam test results of the silicon charge detector for the CREAM cosmic ray balloon mission, Nucl. Instrum. Meth. A535, 158-161, 2004

[22] H. S. Ahn et al., Beam test calibration of the balloon-borne imaging calorimeter for the CREAM experiment, Proc. 11th International Conference on Calorimetry in Particle Physics CALOR2004(Perugia, Italy, 29 March-2 April 2004), Ed. Cecchi, Claudia et al. World Scientific. 532-537, 2005.

[23] J. Wu et al. Monte Carlo Simulations of the ISS-CREAM Instrument, submitted to PoS(ICRC2019).

[24] K. C. Kim et al. On-Orbit Performance of the ISS-CREAM Calorimeter, submitted to PoS(ICRC2019).

[25] R. Takeishi et al. Cosmic-Ray Elemental Spectra Measured With ISS-CREAM, submitted to PoS(ICRC2019).

[26] G. Choi et al. On-Orbit Performance of the ISS-CREAM SCD, submitted to PoS(ICRC2019).

[27] S. Y. Zinn et al., Design, Implementation, and Performance of CREAM Data Acquisition Software, Nuclear Physics B(Poc. Suppl.), 150, 304-307, 2006.

[28] K. C. Kim et al. ISS-CREAM Flight Operations, submitted to PoS(ICRC2019). 